\title{
PHOTOMETRY OF THE GAS-FILLED LAMP ${ }^{1}$
}

\author{
By G. W. Middlekauff and J. F. Skogland
}

\section{CONTENTS}

II. New variables and photometric difficulties................... 588

III. Experimental investigation of the variables................... 589

I. Lamps, apparatus, and methods................. 589

2. Preliminary measurements........................ 590

3. Current variation at constant speed with different voltages..... 590

4. Current variation at constant voltage with different speeds..... 592

5. Voltage variation at constant current with different speeds..... 594

6. Candlepower variation at constant voltage with different speeds. . 595

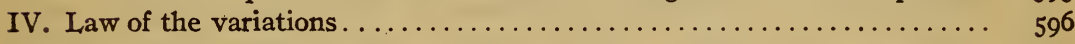

V. Two photometric methods............................ 597

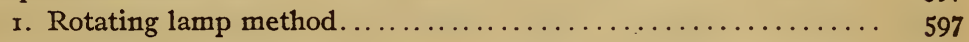

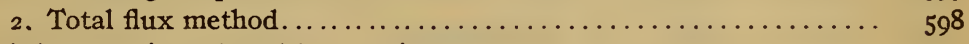

VI. Possible errors introduced by rotation..................... 599

VII. Investigation of the cause of the variations observed............. 600

VIII. A probable explanation of the cause of the variations observed ...... 60 r

IX. Summary $\ldots \ldots \ldots \ldots \ldots \ldots \ldots \ldots \ldots \ldots \ldots \ldots \ldots \ldots \ldots \ldots, 602$

\section{INTRODUCTION}

In a recent paper ${ }^{2}$ the authors have shown that for all vacuum tungsten lamps, within a wide range of wattage, the voltagecurrent-candlepower characteristics are the same regardless of the make or method of manufacture. When this investigation was extended to include gas-filled lamps, it was soon discovered that before consistant and reproducible results could be obtained on the photometer, new and unexpected variables, not present in the vacuum lamp, had to be carefully considered. It is with an experimental study of these variables that this paper is particularly concerned.

\footnotetext{
${ }^{1}$ A preliminary paper on this subject was read at a meeting of the scientific staff, Bureau of Standards, Nov, 20, 1914, and afterwards published in the Electrical World, Dec. 26, I914, p. 1248.

${ }^{2}$ Middlekauff and Skogland, Characteristic Equations of Tungsten Filament Lamps and Their Applica. tion in Heterochromatic Photometry, this Bulletin, 11, p. 483 (Scientific Paper No. 238).
} 


\section{NEW VARIABLES AND PHOTOMETRIC DIFFICULTIES}

In the.gas-filled lamp the filament is coiled into the form of a closely wound spiral or helix, the diameter of which, though small, is large in comparison with the filament itself. Hence, as viewed from certain directions perpendicular to the axis of the lamp, one section of this helical filament may, more or less completely, cut off the light from another section. In addition to this cause of irregularity in the light distribution, there is usually a lack of symmetry in the arrangement of the filament as a whole about the vertical axis of the lamp. Consequently, the light on the photometer screen flickers excessively when the lamp is rotated at ordinary speeds. Hence, without auxiliary apparatus, accurate measurements of mean horizontal candlepower are practically impossible; the weight of the lamps, especially of the larger units, the necessity of using mercury in the rotator, etc., preventing a sufficient reduction of the flicker by the use of high speeds of rotation. Fortunately, this difficulty is readily overcome by placing back of the lamp two mirrors ${ }^{3}$ inclined to each other so that the photometer screen is illuminated simultaneously by the direct light from the lamp and the reflected light from the mirrors. With this arrangement very low speeds of rotation may be used.

Although this expedient obviates the flicker difficulty, it does not eliminate the most serious trouble caused by rotation. It was found that at constant voltage both current and candlepower have different values when the lamp is rotating than when it is stationary, the current changing in one direction and the candlepower always in the opposite direction. In other words, there is a change in the operating efficiency of the lamp although the voltage remains the same. ${ }^{4}$ It was found, further, that the values of current and candlepower may be either increased or decreased by rotation, depending upon the speed, and by amounts sufficiently great to affect seriously the result of candlepower and efficiency determinations.

3 This Bulletin, 2, p. 416; 1906.

1 This general effect was observed about the same time also at the Electrical Testing Laboratories, New York, and described by Dr. C. H. Sharp, Trans. Illum. Eng. Soc., 9, p. Io2r; r9r4. 
The gas-filled lamp therefore presents a new problem in photometry, involving not only greater difficulties on account of greater color difference over those encountered in photometering vacuum lamps but also the difficulty of dealing with variables which, if not properly considered, may introduce considerable uncertainty in the results.

Below are given some experimental results obtained in a systematic study of the lamp and from these the law of the variations is ceduced. From a consideration of this law a practical method of measuring mean horizontal candlepower free from the errors caused by rotation is discovered and an explanation of the cause of the variations observed is offered.

\section{EXPERIMENTAL INVESTIGATION OF THE VARIABLES}

\section{LAMPS, APPARATUS, AND METHODS}

The lamps used in the principal experiments described below were of the nitrogen-filled 450-watt series and 750 -watt and Iooo-watt multiple types, all having round bulbs with the filaments centrally mounted. The number of anchor wires in the different lamps varied from 5 to 9 . Additional experiments were made with a number of 200 -watt series and 750-watt multiple lamps with straight side bulbs, the former having one anchor wire, the latter five. Other experiments were made with an ordinary vacuum lamp filled with nitrogen and also with a vacuum lamp having a coiled filament such as used in the gas-filled lamp.

Measurements of voltage and current were made simultaneously by means of two potentiometers. The rotator was supplied with mercury-cup connections and two sockets so that the lamp could be mounted either tip up or tip down. The rotating mechanism was under perfect control at every speed, which was determined by a specially designed indicator, and the current to the lamp was supplied from a storage battery, thus insuring perfectly steady working conditions. In the candlepower measurements use was made of the two-mirror arrangement described above.

A few rough preliminary tests indicated that for any change in speed the percentage change in candlepower was at least 
several times the percentage change in current but always in the opposite direction. As current can be measured with considerably higher accuracy than candlepower, such measurements furnish a convenient method of determining approximately the variation of the candlepower. It was therefore decided that before making careful determinations of candlepower, it would be advisable to make a series of measurements of voltage, current, and speed, varying only one of these quantities at a time.

\section{PRELIMINARY MEASUREMENTS}

The general effect of rotation on current and power consumption with voltage constant was first determined in three series of fairly accurate measurements made on a II6-volt, 750-watt, short-necked lamp at 90, I05, and II6 volts, respectively. In each series current readings were taken at different predetermined speeds, a reading with the lamp stationary being taken as a check between the changes of speed. During these measurements the lamp was used first tip up and then tip down. With the lamp in either position and the speed above 40 revolutions per minute the current was less than with the lamp stationary and decreased with increasing speed, the effect with tip up being about twice as great as with tip down. Other lamps of the same size and of different sizes (450 watts to rooo watts) and of different manufacture were tested in the same manner and similar results were obtained in every case.

In these preliminary measurements it was learned that very consistent and reproducible current values could be obtained provided the speed of rotation is accurately known and properly controlled; otherwise apparently inconsistent and erratic results were to be expected. Hence, in all subsequent tests the speed was as carefully measured and controlled as the voltage and current.

\section{CURRENT VARIATION AT CONSTANT SPEED WITH DIFFERENT VOLTAGES}

The next step taken was to determine with great care the changes in current due to rotation at a specified speed at different values of voltage through a wide range. This test was applied 
to two lamps, the 750-watt lamp mentioned above and a rooowatt lamp, both rated at II 6 volts. Readings of current corresponding to a chosen voltage were first made with the lamp stationary, then with it rotating at a speed of $150 \mathrm{rpm}$. In this way

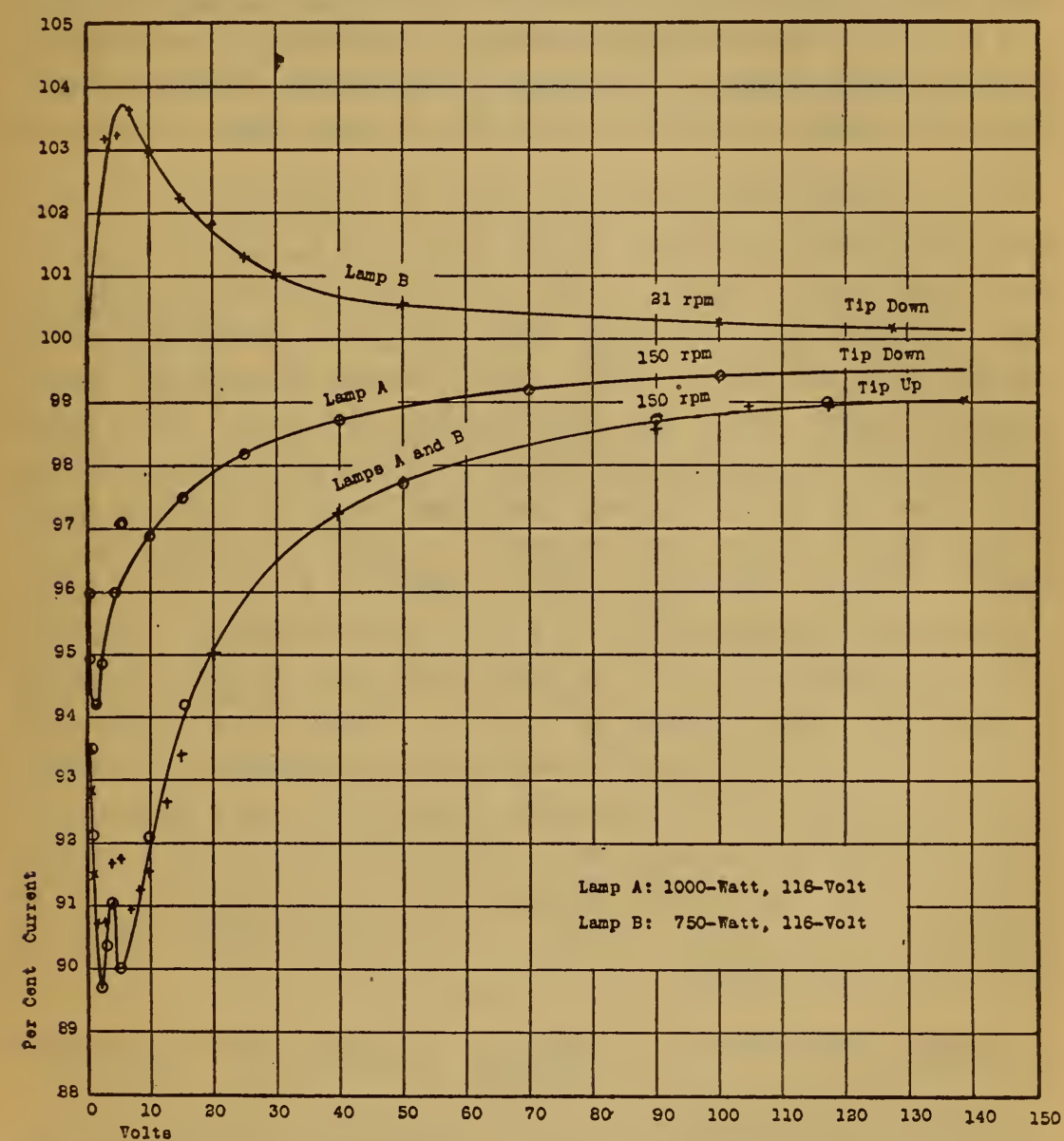

FIG. I.-Ratio of current with lamp rotating at constant speed to current with lamp stationary

the changes in current for each lamp at different voltages over a range from 0.5 volt to roo volts were obtained.

The results for both lamps rotated tip up are shown by the lowest curve of Fig. I, values obtained for the 750-watt lamp 
being represented by crosses and those for the rooo-watt lamp by circles. The behavior of the current in these two lamps is thus shown to be practically identical at every value of voltage except from 3 volts to 8 volts, which is a critical region. It is probable that even within this region these curves would be found to coincide also were measurements made at a sufficiently large number of neighboring points. The upward indentation in the curve at this point is as real and reproducible as any other part of the curve.

Upon rotating these lamps tip down, it was found that for the same speed the percentage change in current for all voltages was only about half as great as when the lamps were rotating tip up. The values for the 1000-watt lamp tip down are given by the middle curve of Fig. I.. This curve is traced through all values obtained except one in the neighborhood of 5 volts. This value indicates that an indentation would probably have been found in this curve also had sufficient time been taken to pass by small voltage steps through this region. It might be stated that, in order to obtain sufficiently steady conditions for accurate measurements of current in this region, a period of at least ro minutes was required at each point, as small variations of speed resulted in relatively large changes in current. These two curves are similar in form, both becoming asymptotic to the axis of abscissas at about I per cent and 0.5 per cent, respectively, below the current observed for the lamp stationary. That is, for this speed, at about 85 per cent of the normal voltage, the current reaches a value which from that point on bears a practically constant ratio to the value at zero speed.

\section{CURRENT VARIATIONS AT CONSTANT VOLTAGE WITH DIFFERENT SPEEDS}

In order to investigate further the behavior of the current in the critical region above mentioned, as well as at other points, measurements of current at constant voltage, with variable speed, in succession at different values of voltage were made on the rooo-watt lamp through a considerable range extending as low as 0.5 volt. The speed was varied from $5 \mathrm{rpm}$ to $200 \mathrm{rpm}$, the lamp being rotated tip down. The results of this test are shown in 
Fig. 2. The important fact indicated by these curves is that as the speed is increased from zero the current, at constant voltage, first increases to a maximum and then decreases to the stationary value, and continues decreasing below the stationary value as the speed is farther increased. (By stationary value is meant the value obtained with the lamp stationary.)

In order to confirm this change in sign of the current variation the 750-watt lamp was tested tip down while rotating at a speed

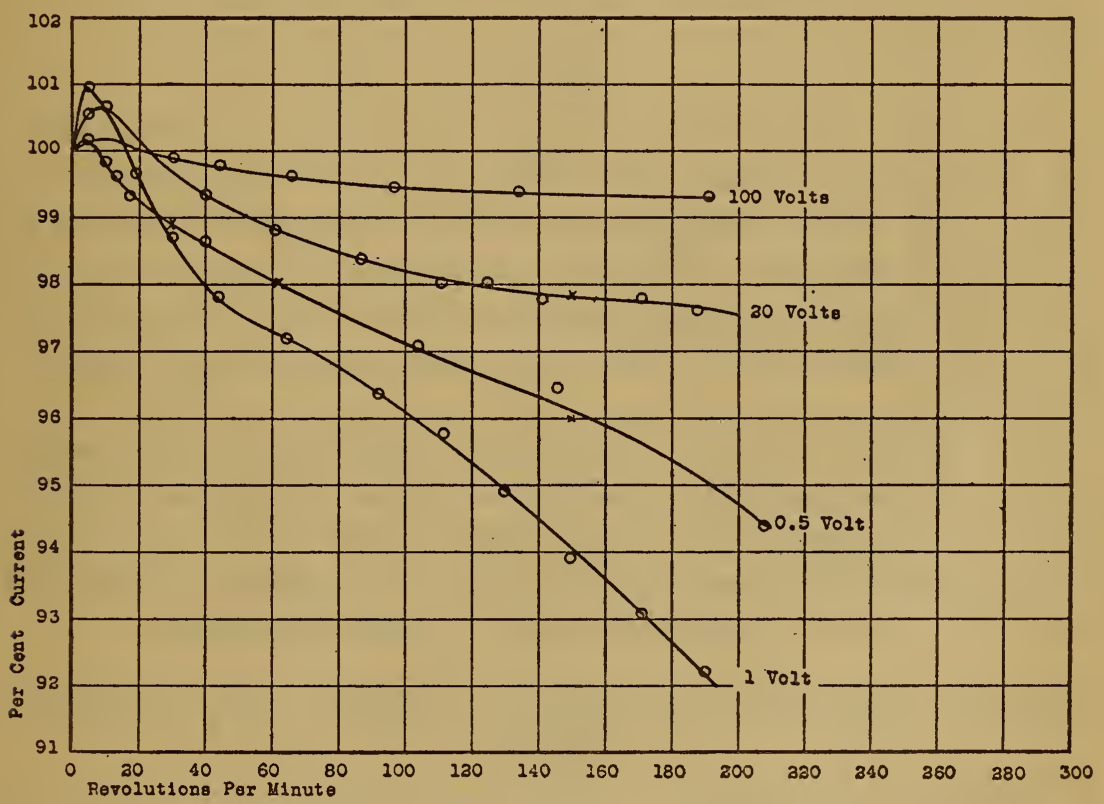

FIG. 2.-Ratio of current with lamp rotating tip down at different speeds to current with lamp stationary; voltage constant at different values

of $2 \mathrm{I} \mathrm{rpm}$. The results of this test are shown by the uppermost curve of Fig. I. It will be noted that this curve is of practically the same form as the other two curves of this figure, but that it is turned in the opposite direction and lies entirely above the roo per cent or stationary values. That is, at this speed of rotation the current is higher than at zero speed for all values of voltage. Hence, correct qualitative results may be obtained at any practical voltage. 
Upon carefully testing ail available 450-watt to Iooo-watt lamps, including both series and multiple types in round bulbs and one 750 -watt lamp in a straight-side bulb, it was found that in every lamp while rotating with the tip down the current at constant voltage returned to the stationary value at a speed between $33 \mathrm{rpm}$ and $40 \mathrm{rpm}$. This held true through a considerable range of voltage, extending above and below the normal, or rated, value. The series lamps included in this test had the same form of mount and practically the same arrangement of the filament as the multiple lamps.

For 200-watt, 6.6-ampere lamps, with $V$-shaped filament and a single anchor wire, it was found necessary to use a speed of from I 40 to $160 \mathrm{rpm}$ to obtain the stationary value of the current, the value of the current being greater than the stationary value up to the speed mentioned. It appears, therefore, that the smaller the number of loops and corresponding anchor wires in the lamp the greater the speed that is required to give the stationary value of the current.

Although there was a considerable difference in the speeds required for lamps having different numbers of loops in the filament, it was possible to find for every lamp tested a particular speed at which the current returned to the stationary value, the speed being in every case such as can be used in practice.

\section{VOLTAGE VARIATION AT CONSTANT CURRENT WITH DIFFERENT SPEEDS}

The effect of rotation on voltage was determined by testing a few of the 450-watt lamps, tip down, with the current held constant at 6.6 amperes and the speed varied over a wide range, beginning at $5 \mathrm{rpm}$ as the lower limit. For every lamp it was found that as the speed was increased from zero the voltage first decreased to a minimum and then increased to the stationary value and continued increasing above the stationary value as the speed was further increased. In other words, the effect of rotation upon the voltage at constant current was opposite in sign to the effect upon the current in the same lamps at constant voltage. 
Since the variable quantity (i. e., voltage or current) in either case returns to its stationary value, it is evident that the speed at which this occurs, and therefore at which the power consumption is the same as when the lamp is stationary, may be determined by holding either quantity (voltage or current) constant and observing the variation of the other as the speed is gradually increased from zero. Principally as a matter of convenience, therefore, all subsequent tests were made at constant voltage.

\section{CANDLEPOWER VARIATION AT CONSTANT VOLTAGE WITH DIFFERENT SPEEDS}

The effect of rotation on candlepower was very strikingly shown by the following experiment: The voltage was so adjusted that the filament when stationary was just visible in a darkened room. Upon rotating the lamp at a speed of about $180 \mathrm{rpm}$ it was found that, although the current decreased in value, the intensity of the light largely increased, the effect being greater with the tip up than with the tip down, thus corroborating the preliminary measurements of current and candlepower at higher voltages. With the lamp in either position and either rotating or stationary the upper loops of the filament glowed more brightly than the lower ones, thereby indicating a considerable difference in temperature between closely neighboring regions of the gas.

Since there was a speed at which the power consumption was the same as when the lamp was stationary, it was considered probable that at this same speed the candlepower also would return to the stationary value. This led to a series of simultaneous measurements of current and relative values of the candlepower, with voltage constant and speed varied over a wide range.

Results obtained in this way with the 750-watt lamp operated at 90 volts are shown by the curves of Fig. 3. All values of current and candlepower, respectively, are expressed in percentages of the value of each obtained with the lamp stationary and the tip down. In determining the candlepower with the lamp stationary, readings were taken at intervals of $7.5^{\circ}$ about the vertical axis, there being a total of 48 determinations.

The curves for the lamp with the tip down are the result of two independent series of measurements made on different days, 
values obtained in the two series being represented by crosses and circles, respectively. The agreement of these two series shows that current and candlepower values are reproducible to a high degree of precision.

The ratio of the ordinates to which these curves were drawn was purposely so chosen as to clearly represent to the eye the constant relation of candlepower to current throughout the whole range of speed employed.

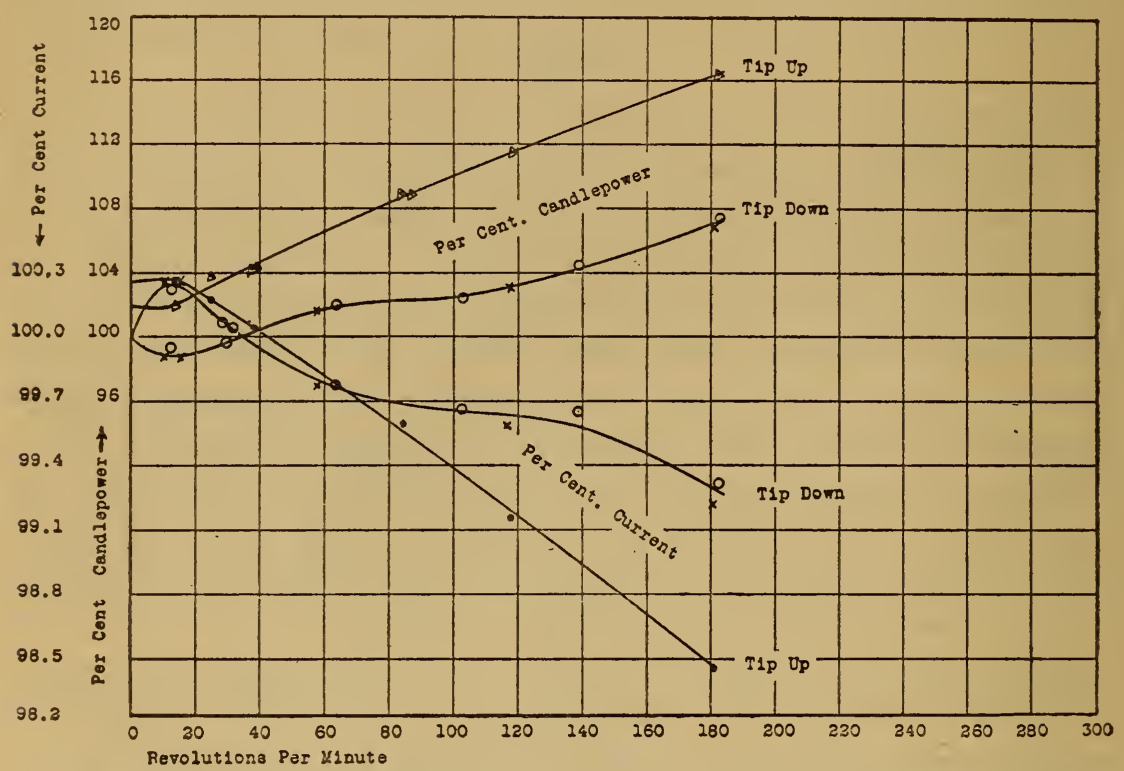

FIG. 3.-Ratio of current and candlepower with lamp rotating tip up and also tip down to current and candlepower, respectively, with lamp stationary tip down

\section{LAW OF THE VARIATIONS}

The results of these experiments may be summarized as follows, in which the voltage is to be regarded as constant:

I. With the lamp stationary, both current and candlepower have higher values when the lamp is mounted tip up than when it is mounted tip down.

2. Rotation of the lamp causes both current and candlepower to change in value but in opposite directions, the change in both being about twice as great when the tip is up as when it is down. 
3. As the speed is increased from zero the current first increases to a maximum and then returns to the value it has when the lamp is stationary and continues decreasing below this value as the speed is further increased. The candlepower varies in the opposite direction.

4. For each position of the lamp there is a particular speed at which both current and candlepower have each the same value as when the lamp is stationary, the speed at which this occurs being practically the same for all lamps having the same number of loops in the filament. For lamps having different forms of filament mounting the speed for the above condition varies from lamp to lamp, being highest for those having the smallest number of loops.

\section{TWO PHOTOMETRIC METHODS}

\section{ROTATING LAMP METHOD}

From a consideration of the above results it is evident that if the voltage be held constant and the lamp be rotated at the speed at which the current has the same value as when the lamp is stationary an accurate determination of mean horizontal candlepower may be made and the same efficiency rating given to the lamp as it would have if operated stationary at the same voltage. It is fortunate that in the position in which gas-filled lamps are most generally used in practice-that is, tip down-the speed for the above condition is somewhat higher and therefore more practical for most lamps than the speed for the same condition with the tip up.

Although the above method has its limitations which are stated below, it fulfills all the requirements for determining the voltage-current-candlepower characteristics of gas-filled lamps by the use of an ordinary bar photometer outfit. With a knowledge of the reduction factor, the watts per candle of the lamp may be determined for any given voltage, and then with the lamp stationary and with this voltage as a starting point the variations of current, candlepower, and watts per candle with voltage are as readily determined for a gas-filled lamp as for one of the vacuum type. In watts per candle determinations the factor expressing the relation of mean spherical to horizontal 
candlepower in the given direction must, of course, be applied to the candlepower readings.

For groups of lamps having the same form of mounting, and therefore the same reduction factor, the method of rotation is perfectly satisfactory in determining relative candlepower and efficiency ratings, and practically the same speed may be used for all lamps of the group. However, as stated above, this method presupposes a knowledge of the reduction factor, which for gas-filled lamps in general, as now made in the present more or less experimental stage of manufacture, varies considerably from lamp to lamp, principally on account of the great variety of methods of mounting the filament.

\section{TOTAL FLUX METHOD}

During life tests of vacuum lamps the blackening of the bulb in all directions is about proportional to the distribution of the light, and hence a measure of the reduction in light during life may be obtained by observing the reduction in the mean horizontal candlepower. In the gas-filled lamp, however, the greater proportion of the blackening occurs at the top of the bulb, the volatilized material thrown off from the filament being carried upward by the gas, and hence a true measure of the reduction in light during the life of the lamp can not be obtained by mean horizontal candlepower measurements alone but by determinations of the total flux or mean spherical candlepower. This can be most conveniently done by means of an integrating photometer, such as the Ulbricht sphere. The advantage of the sphere over other forms of integrating photometers, such as the Matthews instrument, in measuring gas-filled lamps is that the lamps are measured while stationary and thus the complications arising from rotation are avoided.

For the determination of the voltage-current-candlepower characteristics the sphere has no particular advantage over the method of rotation and has the possible disadvantage in that if the coating of the sphere is not perfectly nonselective the ratio of the candlepower at different voltages may be more or less affected by a change in the absorption of the light at the different colors. 


\section{POSSIBLE ERRORS INTRODUCED BY ROTATION}

The accompanying table shows the errors possible in efficiency determinations and the consequent errors in life values introduced by measuring candlepower and watts while the lamp is rotating. The values given are the result of photometric measurements made at different speeds on a 750-watt, I I 5-volt, nitrogen-filled lamp having nine anchor wires supporting the filament.

Data on Errors in Efficiency and Life Values

\begin{tabular}{r|r|r|r|r|r|r}
\hline Position of lamp & $\begin{array}{c}\text { Speed in } \\
\text { rpm }\end{array}$ & $\begin{array}{c}\text { Rated volts } \\
\text { for 0.70 } \\
\text { watt per } \\
\text { candle, } \\
\text { lamp } \\
\text { rotating }\end{array}$ & $\begin{array}{c}\text { Watts per } \\
\text { candle at } \\
\text { rated volts; } \\
\text { lamp sta- } \\
\text { tionary, tip } \\
\text { down }\end{array}$ & $\begin{array}{r}\text { Per cent } \\
\text { error in } \\
\text { rated } \\
\text { volts }\end{array}$ & $\begin{array}{r}\text { Per cent } \\
\text { error in } \\
\text { rated watts } \\
\text { per candle }\end{array}$ & $\begin{array}{c}\text { Per cent } \\
\text { vife at rated } \\
\text { volts; lamp } \\
\text { stationary, } \\
\text { tip down }\end{array}$ \\
\hline 0 & 115.0 & 0.700 & 0.0 & 0.0 & 100 \\
35 & 115.0 & 0.700 & 0.0 & 0.0 & 100 \\
70 & 113.5 & 0.718 & 1.3 & 2.6 & 121 \\
120 & 112.8 & 0.726 & 1.9 & 3.7 & 131 \\
180 & 109.9 & 0.760 & 4.4 & 8.6 & 184 \\
0 & 113.8 & 0.713 & 1.0 & 1.9 & 115 \\
14 & 113.8 & 0.713 & 1.0 & 1.9 & 115 \\
70 & 110.1 & 0.757 & 4.3 & 8.1 & 178 \\
120 & 107.4 & 0.793 & 6.6 & 13.3 & 252 \\
180 & 104.4 & 0.837 & 9.2 & 19.6 & 375 \\
\hline
\end{tabular}

On account of the increased efficiency of this lamp caused by rotation, for example, at $70 \mathrm{rpm}$ tip down, it was found to have a specific consumption of 0.7 watt per candle at II3.5 volts instead of at II 5 volts as found at zero speed with the tip down. If, therefore, the lamp had been photometered at the factory while rotating at this speed, it would have been rated at I I 3.5 volts and would have been operated on the life rack at 0.718 watt per candle instead of 0.7 watt per candle and would have had a life 2 I per cent greater than would be expected from the rating. If, however, it had been photometered with the tip up at $\mathrm{I} 80 \mathrm{rpm}$ (as is sometimes done in practice), it would have been rated as having a specific consumation of 0.7 watt per candle at 104.4 volts. If put upon the life rack at this voltage, it would operate at 0.837 watt per candle instead of 0.7 watt per candle and give a life 275 per cent in excess of normal. In other 
words, a long-life performance is fictitious at the given specific consumption rating, or the consumption rating is fictitious for the given life. This holds true if the specific consumption is determined at any speed above that at which the current and candlepower have the same value as when the lamp is stationary with the tip down. For this lamp the particular speed is 35 rpm. For speeds below this value with the tip down, the specific consumption rating would be too high and the life value would be lower than expected from the rating.

\section{INVESTIGATION OF THE CAUSE OF THE VARIATIONS OBSERVED}

The following experiments were made with a view to finding the cause of the variations observed in this type of lamp:

I. To determine the effect of increased external ventilation of the bulb caused by rotation, a draft of air was driven against a I000-watt lamp by means of an electric fan and a series of measurements of current at constant voltage were made at different speeds with "air on" and "air off." It was found that at every speed the cooling effect of the air caused a small but measurable increase in the current but that this increase was very small in comparison with the decrease in current caused by the rotation of the lamp. Hence, the decrease in current can not be attributed to an increase in external ventilation of the bulb by rotation.

2. Another test was made to determine whether or not the earth's magnetic field had a noticeable effect in producing a counter electromotive force, thus changing the effective voltage on the lamp. This was done by placing the lamp in the middle of a solenoid having a field strength of about 500 gausses, and the current in the lamp was observed when the current in the solenoid was thrown on and off, both with the lamp stationary and rotating. As no change was detected it was concluded that the much weaker field of the earth certainly did not produce a counter emf of sufficient magnitude to account for any part of the changes noted.

3. To determine the effect of possible distortion of the filament upon the candlepower in the horizontal plane, candlepower measurements at various angles with the vertical were made upon the 
Iooo-watt lamp (lamp A, figs. I and 2) rotating with the tip down, first at a speed of $45 \mathrm{rpm}$ and then at a speed of $200 \mathrm{rpm}$. There was practically the same percentage increase in candlepower at every angle when the lamp was rotating at the higher speed-that is, the spherical reduction factor, which was about 0.86 , was not appreciably changed by rotation. Hence distortion of the filament was not the cause of the changes observed.

4. A roo-watt vacuum tungsten lamp having a coiled filament similar to that of the gas-filled lamp, and similarly mounted in the bulb, when rotated exhibited none of the changes observed in the case of the gas-filled lamp. The same was found to be true for a 500 -watt vacuum tungsten lamp having an ordinary (not coiled) filament mounted in the usual manner. On the other hand an ordinary 250 -watt lamp when filled with nitrogen exhibited all the variations of the coiled filament gas-filled. lamp, the current at constant voltage returning to the stationary value at a speed of about $100 \mathrm{rpm}$. It was therefore concluded that the gas alone must be the disturbing element which causes the changes observed in the gas-filled lamp.

\section{A PROBABLE EXPLANATION OF THE CAUSE OF THE VARIATIONS OBSERVED}

The results of the above tests show quite conclusively that practically the whole of the effect observed in the change in current, candlepower, and specific consumption arises from a change in the convection currents of the gas within the bulb. At low speeds, as seen above, there is an increase in the current over the stationary value for all voltages, the greater percentage increase being at the low voltages. At high speeds there is a decrease in current as compared with the stationary value for all voltages, the greatest percentage decrease being also at low voltages.

When the lamp is stationary there is a considerable difference in temperature between the gas at the top and bottom of the bulb. Very low speeds of rotation disturb the steady current of hot gas which is rising in the center and flowing down at a distance from the axis. The result is an increase in heat convection by the gas, and consequently a lowering of the temperature of the filament. 
As the filament has a positive temperature-resistance coefficient, the resistance decreases and consequently the current increases at constant voltage. At high values of voltage the mean temperature of the gas is higher than at low voltage, consequently the temperature of the filament is reduced in a lesser degree by rotation and the change in current, though positive, is less at high than at low voltage.

On the other hand, when the lamp is rotated at high speeds the cooler gas at the bottom of the bulb is thrown out by centrifugal force, which varies as the square of the speed, and the hot gas tends to remain near the center, thus considerably retarding the convection currents which cool the filament. The result is an increase in the resistance of the filament as a whole and a decrease in the value of the current. At high voltages the mean temperature of the gas is higher than at low voltage and the retarding effect due to centrifugal force is decreased; hence the change in current due to rotation is less than at low voltages.

At low speeds, as seen above, the temperature of the filament is lowered and consequently the candlepower is decreased. At high speeds the temperature of the filament is raised, and hence there is an increase in the candlepower.

\section{SUMMARY}

The new high efficiency gas-filled lamp introduces variables not hitherto encountered in the photometry of incandescent electric lamps. On account of the comparative broadness of the filament spiral and the dissymmetry of the filament mounting, there is considerable irregularity in the distribution of the light about the vertical axis. Consequently, when the lamp is rotated, as is commonly done in rating lamps at the factory, the light as seen in the photometer flickers so excessively as to render accurate measurements of candlepower practically impossible without the use of auxiliary apparatus. However, as is sometimes done, if two mirrors inclined to each other be placed back of the lamp, the flickering is so much reduced as to permit accurate candlepower measurements even at very low speeds of rotation. 
But this expedient does not eliminate the most serious trouble caused by rotation. It was found that at constant voltage both the current consumed and the candlepower are different when the lamp is rotating than when it is stationary, the current changing in one direction and the candlepower always in the opposite direction; that is, there is a change in the operating efficiency of the lamp. Furthermore, this change in efficiency may be either positive or negative, depending upon the speed, and it is about twice as great when the lamp is rotating tip up as when it is rotating tip down.

Fortunately, from the standpoint of photometry, there is for each lamp in either position a particular speed at which the current and the candlepower have the same values, respectively, as when the lamp is stationary. Hence, with the lamp rotating at this speed its candlepower can be measured with accuracy in spite of its rotation. The speed for the above condition is practically the same for all lamps having the same number of loops in the filament; but for lamps having different forms of filament mounting it varies from lamp to lamp, being greatest for those having the smallest number of loops in the filament.

If the above precaution as to speed adjustment is not observed and lamps are rated while rotating at speeds ordinarily used in photometering vacuum lamps, the errors which enter may amount to as much as I to 2 per cent in current, or watts, in one direction, and as much as $\mathrm{I} 5$ to 20 per cent in candlepower in the opposite direction. Hence the voltage found for a desired operating efficiency may be so much in error as to give a lamp on test at this rated voltage a fictitious life value three or four times as large as the lamp would give if it were operated stationary at a voltage corresponding to that efficiency which during the rating was only apparent. That is, the lamp may be given credit for a much longer life than it really deserves. On the other hand, the speed may be such as to cause errors in the opposite direction resulting in a lamp life much shorter than would be expected from the apparent efficiency rating.

Another peculiarity of the gas-filled lamp is that while it burns the blackening occurs, not all over the bulb in approximate pro- 
portion to the light distribution as in the vacuum lamp, but principally at the top of the bulb because the volatilized material is carried upward by the gas. Hence in making a life test a true measure of the reduction in total light during the life of the lamp can not be obtained, in the usual manner, by mean horizontal candlepower measurements, but by determinations of the total flux or mean spherical candlepower. This is accomplished most rapidly and conveniently by means of an integrating photometer, such as the Ulbricht sphere, in which the lamp is measured stationary, and thus all the complications arising from rotation are entirely avoided.

As to the cause of the variations observed in candlepower and efficiency when the lamp is rotated, it is concluded from the results of a number of special tests that the whole effect is produced by a change in the convection currents of the gas, a consequent variation in the temperature distribution in the bulb, resulting in a change in the resistance, and therefore a variation in the current and candlepower of the lamp.

The authors acknowledge their indebtedness to Dr. E. B. Rosa for his kindly interest and valuable suggestions, and to $\mathrm{H}$. B. Sinelnick for efficient assistance in the laboratory work.

WASHINGTON, January 25, I9I 5. 